\title{
Starting solutions for the motion of a generalized Burgers' fluid between coaxial cylinders
}

Muhammad Jamil ${ }^{1,2^{*}}$ and Constantin Fetecau ${ }^{1,3}$

\author{
* Correspondence: jqrza26@yahoo. \\ com \\ ${ }^{1}$ Abdus Salam School of \\ Mathematical Sciences, GC \\ University, Lahore, Pakistan \\ Full list of author information is \\ available at the end of the article
}

\begin{abstract}
The unsteady flow of a generalized Burgers' fluid, between two infinite coaxial circular cylinders, is studied by means of the Laplace and finite Hankel transforms. The motion of the fluid is produced by the inner cylinder that, after the initial moment, applies a longitudinal time dependent shear to the fluid. The solutions that have been obtained, presented in series form in terms of usual Bessel functions, satisfy all imposed initial and boundary conditions. Moreover, the corresponding solutions for Burgers' fluids appear as special cases of present results. For large values of $t$, these solutions are going to the steady solutions that are the same for both kinds of fluids. Finally, the influence of the material parameters on the fluid motion, as well as a comparison between models, is shown by graphical illustrations.

Mathematics Subject Classification (2010). 76A05; 76A10.

Keywords: Generalized Burgers' fluid, coaxial cylinders, velocity field, time-dependent shear stress, Laplace and Hankel transforms
\end{abstract}

\section{Introduction}

Recently, considerable attention has been focused to study the behavior of non-Newtonian fluids. Many substances of multi-phase nature and/or of high molecular weight are frequently encountered in disciplinary fields such as chemical engineering, food stuff, bio-medicine and so forth, and are also closely related to industrial processes. Typical non-Newtonian characteristics include shear-thinning, shear-thickening, viscoelasticity, viscoplasticity (i.e., the exhibition of an apparent yield stress) and so on. In particular, polymer melts and solutions, liquid crystals or biological fluids exhibit such properties which lead to non-linear viscoelastic behavior that cannot be simply described by the classical Navier-Stokes theory. Non-Newtonian fluids form a broad class of fluids in which the relation connecting the shear stress and shear rate is nonlinear and hence there is no universal constitutive model available which exhibits the characteristics of all non-Newtonian fluids. Moreover, due to the flow behavior of these fluids, the governing equations become more complex to handle as additional non-linear terms appear in the equations of motion. Numerous models have been proposed to describe the response corresponding to non-Newtonian fluids. They are usually classified as fluids of differential, rate and integral type. Amongst the non-Newtonian fluids, the rate type fluids are those which take into account the elastic and memory effects. The simplest subclasses of rate type fluids are those of Maxwell and

(c) 2012 Jamil and Fetecau; licensee Springer. This is an Open Access article distributed under the terms of the Creative Commons Attribution License (http://creativecommons.org/licenses/by/2.0), which permits unrestricted use, distribution, and reproduction in any medium, provided the original work is properly cited. 
Oldroyd-B fluids. But these fluid models do not exhibit rheological properties of many real fluids such as asphalt in geomechanics and cheese in food products.

Recently, a thermodynamic framework has been put into place to develop the 1D rate type model known as Burgers' model [1] to the frame-indifferent 3D form by Krishnan and Rajagopal [2]. This model has been successfully used to describe the motion of the earth's mantle. The Burgers' model is the preferred model to describe the response of asphalt and asphalt concrete [3]. This model is mostly used to model other geological structures, such as Olivine rocks [4] and the propagation of seismic waves in the interior of the earth [5]. In the literature, the vast majority of the flows of the rate type models has been discussed using Maxwell and Oldroyd-B models. However, the Burgers' model has not received much attention in spite of its diverse applications. We here mention some of the studies [6-12] made by using Burgers' model. The most of the above mentioned studies dealt with problems in which the velocity is given on the boundary. The motion of a fluid due to translating or rotating cylinder is of great interest to both theoretical and practical domains. The first exact solutions for motions of non-Newtonian fluids due to a circular cylinder that applies a longitudinal or rotational shear stress to the fluid are those of Bandelli and Rajagopal [13, Sects. 4 and 5]. These solutions, for rotational shear stresses, have been recently extended to generalized Burgers fluids [11,12].

The aim of this note is to extend the results of Bandelli and Rajagopal [13, Sect. 4] to Burgers and generalized Burgers fluids. More exactly, we study the motion of a generalized Burgers' fluid between two infinite coaxial circular cylinders. The motion of the fluid is induced by the inner cylinder that applies a longitudinal time dependent shear to the fluid. The solutions that have been obtained satisfy both the governing equations and all imposed initial and boundary conditions. They can immediately be reduced to the similar solutions for Burgers' fluids. As a check of the results, the equivalence of general solutions (for small values of the material parameters $\lambda_{2}$ and $\lambda_{4}$ ) with the known solutions for Oldroyd-B fluids is shown by graphical illustrations. Furthermore, in order to reveal some relevant physical aspects of these results, the diagrams of the velocity and shear stress are depicted against $r$ for different values of pertinent parameters.

\section{Basic governing equations}

The equations governing the flow of an incompressible fluid include the continuity equation and the momentum equation. In the absence of body forces, they are

$$
\nabla \cdot \mathbf{V}=0, \quad \frac{\partial \mathbf{V}}{\partial t}+(\mathbf{V} \cdot \nabla) \mathbf{V}=\frac{1}{\rho}(\nabla \cdot \mathrm{T}),
$$

where $\rho$ and $\mathbf{V}$ are, respectively, the fluid density and velocity vector and $\nabla$ represents the gradient operator.

The Cauchy stress tensor $\mathbf{T}$ for an incompressible generalized Burgers' fluid is characterized by the following constitutive equations [10-12]

$$
\mathbf{T}=-p \mathbf{I}+\mathbf{S}, \mathbf{S}+\lambda_{1} \frac{\delta \mathbf{S}}{\delta t}+\lambda_{2} \frac{\delta^{2} \mathbf{S}}{\delta t^{2}}=\mu\left[\mathbf{A}+\lambda_{3} \frac{\delta \mathbf{A}}{\delta t}+\lambda_{4} \frac{\delta^{2} \mathbf{A}}{\delta t^{2}}\right],
$$


where $-p \mathbf{I}$ denotes the indeterminate spherical stress, $\mathbf{S}$ is the extra-stress tensor, $\mathbf{A}=$ $\mathbf{L}+\mathbf{L}^{T}$ is the first Rivlin-Ericksen tensor ( $\mathbf{L}$ being the velocity gradient), $\mu$ is the dynamic viscosity, $\lambda_{1}$ and $\lambda_{3}\left(<\lambda_{1}\right)$ are relaxation and retardation times, $\lambda_{2}$ and $\lambda_{4}$ are new material parameters of the generalized Burgers' fluid (having the dimension of $t^{2}$ ), and $\delta / \delta t$ denotes the upper convected derivative defined by [10]

$$
\frac{\delta \mathbf{S}}{\delta t}=\frac{d \mathbf{S}}{d t}-\mathbf{L S}-\mathbf{S L}^{T}, \quad \frac{\delta^{2} \mathbf{S}}{\delta t^{2}}=\frac{\delta}{\delta t}\left(\frac{\delta \mathbf{S}}{\delta t}\right) .
$$

Into above relation, $d / d t$ is the usual material time derivative.

This model includes as special cases the Burgers' model (for $\lambda_{4}=0$ ), Oldroyd-B model (for $\lambda_{2}=\lambda_{4}=0$ ), Maxwell model (for $\lambda_{2}=\lambda_{3}=\lambda_{4}=0$ ) and the linearly viscous fluid model when $\lambda_{1}=\lambda_{2}=\lambda_{3}=\lambda_{4}=0$. In some special flows, like that to be here considered, the governing equations corresponding to generalized Burgers' fluids also resemble those for second grade fluids. However, closed form expressions for the similar solutions corresponding to Oldroyd-B, Maxwell, second grade and Newtonian fluids cannot be obtained as limiting cases of general solutions.

For the problem under consideration we assume a velocity field $\mathbf{V}$ and an extrastress tensor $\mathbf{S}$ of the form

$$
\mathbf{V}=\mathbf{V}(r, t)=v(r, t) \mathbf{e}_{z \prime} \quad \mathbf{S}=\mathbf{S}(r, t),
$$

where $\mathbf{e}_{z}$ is the unit vector in the $z$-direction of a cylindrical coordinate system $r, \theta, z$. For these flows the constraint of incompressibility is automatically satisfied.

Substituting Eq. (3) into Eq. (2), and assuming that the fluid is at rest up to the moment $t=0$, i.e.:

$$
\mathbf{V}(r, 0)=\frac{\partial \mathbf{V}(r, 0)}{\partial t}=\frac{\partial^{2} \mathbf{V}(r, 0)}{\partial t^{2}}=\mathbf{0}, \quad \mathbf{S}(r, 0)=\frac{\partial \mathbf{S}(r, 0)}{\partial t}=\mathbf{0},
$$

it results that $S_{r r}=S_{r \theta}=S_{\theta \theta}=S_{\theta z}=0$ for all time and [10]

$$
\left(1+\lambda_{1} \frac{\partial}{\partial t}+\lambda_{2} \frac{\partial^{2}}{\partial t^{2}}\right) \tau(r, t)=\mu\left(1+\lambda_{3} \frac{\partial}{\partial t}+\lambda_{4} \frac{\partial^{2}}{\partial t^{2}}\right) \frac{\partial v(r, t)}{\partial r},
$$

where $\tau(r, t)=S_{r z}(r, t)$ is the non-trivial tangential stress. The equations of motion, in the absence of body forces, reduce to [14]

$$
\frac{\partial p}{\partial r}=\frac{\partial p}{\partial \theta}=0 \quad \text { and } \quad-\frac{\partial p}{\partial z}+\frac{\partial \tau(r, t)}{\partial r}+\frac{\tau(r, t)}{r}=\rho \frac{\partial v(r, t)}{\partial t} .
$$

Assuming that there is no applied pressure gradient along the axial direction, Eqs. (5) and (6) lead to the following governing equation for velocity

$$
\left(1+\lambda_{1} \frac{\partial}{\partial t}+\lambda_{2} \frac{\partial^{2}}{\partial t^{2}}\right) \frac{\partial v(r, t)}{\partial t}=v\left(1+\lambda_{3} \frac{\partial}{\partial t}+\lambda_{4} \frac{\partial^{2}}{\partial t^{2}}\right)\left(\frac{\partial^{2}}{\partial r^{2}}+\frac{1}{r} \frac{\partial}{\partial r}\right) v(r, t),
$$

where $v=\mu / \rho$ is the kinematic viscosity of the fluid and $\rho$ is its constant density.

\section{Starting flow due to a time dependent shear stress}

Let us consider an incompressible generalized Burgers' fluid at rest in an annular pipe between two coaxial circular cylinders of radii $R_{1}$ and $R_{2}\left(>R_{1}\right)$. At time $t=0^{+}$, a time dependent longitudinal shear stress 


$$
\tau\left(R_{1}, t\right)=f\left[1-\frac{p_{2} e^{p_{1} t}-p_{1} e^{p_{2} t}}{p_{2}-p_{1}}\right], \quad p_{1}, p_{2}=\frac{-\lambda_{1} \pm \sqrt{\lambda_{1}^{2}-4 \lambda_{2}}}{2 \lambda_{2}},
$$

and $f$ is a constant, is applied to the inner cylinder. Due to the shear, the fluid is gradually moved. Its velocity is of the form (3) while the governing equations are given by Eqs. (5) and (7). The appropriate initial and boundary conditions are

$$
\begin{gathered}
v(r, 0)=\frac{\partial v(r, 0)}{\partial t}=\frac{\partial^{2} v(r, 0)}{\partial t^{2}}=0 ; \quad \tau(r, 0)=\frac{\partial \tau(r, 0)}{\partial t}=0 ; \quad r \in\left(R_{1}, R_{2}\right] \\
\left.\left(1+\lambda_{1} \frac{\partial}{\partial t}+\lambda_{2} \frac{\partial^{2}}{\partial t^{2}}\right) \tau(r, t)\right|_{r=R_{1}}=\left.\mu\left(1+\lambda_{3} \frac{\partial}{\partial t}+\lambda_{4} \frac{\partial^{2}}{\partial t^{2}}\right) \frac{\partial v(r, t)}{\partial r}\right|_{r=R_{1}}=f ; \quad t>0,
\end{gathered}
$$

and

$$
v\left(R_{2}, t\right)=0 ; \quad t>0 .
$$

It is easy to see that $\tau\left(R_{1}, t\right)$ given by Eq. (8) is just the solution of Eq. $(10 a)_{1}$. Furthermore, making $\lambda_{1}, \lambda_{2}$ and $\lambda_{4} \rightarrow 0$, Eq. (10a) reduces to the boundary condition (4.3) used by Bandelli and Rajagopal [13]. It corresponds to a problem with a constant shear stress on a part of the boundary, namely

$$
\tau\left(R_{1}, t\right)=\left.\mu\left(1+\lambda_{3} \frac{\partial}{\partial t}\right) \frac{\partial v(r, t)}{\partial r}\right|_{r=R_{1}}=f, \quad v\left(R_{2}, t\right)=0 ; \quad t>0 .
$$

In order to solve the partial differential equations (7) and (5), with the initial and boundary conditions (9) and (10), we shall use the Laplace and finite Hankel transforms.

\subsection{Calculation of the velocity field}

Applying the Laplace transform to Eq. (7) and having in mind the initial and boundary conditions (9) and (10), we find that

$$
\left(q+\lambda_{1} q^{2}+\lambda_{2} q^{3}\right) \bar{v}(r, q)=v\left(1+\lambda_{3} q+\lambda_{4} q^{2}\right)\left(\frac{\partial^{2}}{\partial r^{2}}+\frac{1}{r} \frac{\partial}{\partial r}\right) \bar{v}(r, q) ; \quad r \in\left(R_{1}, R_{2}\right),
$$

where the image function $\bar{v}(r, q)=\mathcal{L}\{v(r, t)\}$ has to satisfy the conditions

$$
\frac{\partial \bar{v}\left(R_{1}, q\right)}{\partial r}=\frac{f}{\mu} \frac{1}{q\left(1+\lambda_{3} q+\lambda_{4} q^{2}\right)}, \quad \bar{v}\left(R_{2}, q\right)=0 .
$$

In the following, we denote by $[15,16]$

$$
\bar{v}_{H}\left(r_{n}, q\right)=\int_{R_{1}}^{R_{2}} r \bar{v}(r, q) B\left(r, r_{n}\right) d r ; \quad n=1,2,3, \ldots
$$

the Hankel transform of $\bar{v}(r, q)$, where

$$
B\left(r, r_{n}\right)=J_{0}\left(r r_{n}\right) Y_{1}\left(R_{1} r_{n}\right)-J_{1}\left(R_{1} r_{n}\right) Y_{0}\left(r r_{n}\right)
$$

$r_{n}$ are the positive roots of the transcendental equation $B\left(R_{2}, r\right)=0$ and $J_{p}(\bullet)$ and $Y_{p}$ $(\bullet)$ are Bessel functions of the first and second kind of order $p$. Multiplying both sides of Eq. (12) by $r B\left(r, r_{n}\right)$, integrating with respect to $r$ from $R_{1}$ to $R_{2}$ and taking into 
account the conditions (13) and the identity [17, Eq. (19)]

$$
\int_{R_{1}}^{R_{2}} r\left[\frac{\partial^{2} \bar{v}(r, q)}{\partial r^{2}}+\frac{1}{r} \frac{\partial \bar{v}(r, q)}{\partial r}\right] B\left(r, r_{n}\right) d r=\frac{2}{\pi r_{n}} \frac{\partial \bar{v}\left(R_{1}, q\right)}{\partial r}-r_{n}^{2} \bar{v}_{H}\left(r_{n}, q\right),
$$

we find that

$$
\bar{v}_{H}\left(r_{n}, q\right)=\frac{2 f}{\rho \pi r_{n}} \frac{1}{q\left[\lambda_{2} q^{3}+\left(\lambda_{1}+\lambda_{4} v r_{n}^{2}\right) q^{2}+\left(1+\lambda_{3} v r_{n}^{2}\right) q+\nu r_{n}^{2}\right]} .
$$

For a suitable presentation of the final results, we rewrite Eq. (17) in the following equivalent form

$$
\bar{v}_{H}\left(r_{n}, q\right)=\frac{2 f}{\pi \mu r_{n}^{3}} \frac{1}{q}-\frac{2 f}{\pi \mu r_{n}^{3}} \frac{\lambda_{2} q^{2}+\left(\lambda_{1}+\lambda_{4} v r_{n}^{2}\right) q+1+\lambda_{3} v r_{n}^{2}}{\lambda_{2} q^{3}+\left(\lambda_{1}+\lambda_{4} v r_{n}^{2}\right) q^{2}+\left(1+\lambda_{3} v r_{n}^{2}\right) q+v r_{n}^{2}},
$$

and use the known results (see for instance [15-17])

$$
\bar{v}(r, q)=\frac{\pi^{2}}{2} \sum_{n=1}^{\infty} \frac{r_{n}^{2} J_{0}^{2}\left(R_{2} r_{n}\right) B\left(r, r_{n}\right)}{J_{1}^{2}\left(R_{1} r_{n}\right)-J_{0}^{2}\left(R_{2} r_{n}\right)} \bar{v}_{H}\left(r_{n}, q\right), \quad \int_{R_{1}}^{R_{2}} r \ln \left(\frac{r}{R_{2}}\right) B\left(r, r_{n}\right) d r=\frac{2}{\pi R_{1} r_{n}^{3}},
$$

In order to obtain the velocity field $v(r, t)=\mathcal{L}^{-1}\{\bar{v}(r, q)\}$, we apply the inverse Laplace transform to Eq. (18) and use the identities (19) and Eq. (A1) from the Appendix. As a result, we find for the velocity field, the simple expression

$$
\begin{gathered}
v(r, t)=\frac{f}{\mu} R_{1} \ln \left(\frac{r}{R_{2}}\right)+\frac{\pi f}{\rho \lambda_{2}} \sum_{n=1}^{\infty} \frac{r_{n} J_{0}^{2}\left(R_{2} r_{n}\right) B\left(r, r_{n}\right)}{J_{1}^{2}\left(R_{1} r_{n}\right)-J_{0}^{2}\left(R_{2} r_{n}\right)} \\
\times\left[\frac{e^{q_{1 n} t}}{q_{1 n}\left(q_{1 n}-q_{2 n}\right)\left(q_{1 n}-q_{3 n}\right)}+\frac{e^{q_{3 n} t}}{q_{2 n}\left(q_{2 n}-q_{1 n}\right)\left(q_{2 n}-q_{3 n}\right)}+\frac{1}{q_{3 n}\left(q_{3 n}-q_{1 n}\right)\left(q_{3 n}-q_{2 n}\right)}\right],
\end{gathered}
$$

where

$$
q_{\text {in }}=s_{\text {in }}-\frac{\lambda_{1}+\lambda_{4} v r_{n}^{2}}{3 \lambda_{2}}, i=1,2,3 .
$$

In above relations (see the Cardano's formulae [18])

$$
\begin{aligned}
& s_{1 n}=\sqrt[3]{-\frac{\beta_{1 n}}{2}+\sqrt{\frac{\beta_{1 n}^{2}}{4}+\frac{\alpha_{1 n}^{3}}{27}}+\sqrt[3]{-\frac{\beta_{1 n}}{2}-\sqrt{\frac{\beta_{1 n}^{2}}{4}+\frac{\alpha_{1 n}^{3}}{27}}}} \\
& s_{2 n}=\omega \sqrt[3]{-\frac{\beta_{1 n}}{2}+\sqrt{\frac{\beta_{1 n}^{2}}{4}+\frac{\alpha_{1 n}^{3}}{27}}}+\omega^{2} \sqrt[3]{-\frac{\beta_{1 n}}{2}-\sqrt{\frac{\beta_{1 n}^{2}}{4}+\frac{\alpha_{1 n}^{3}}{27}}}, \\
& s_{3 n}=\omega^{2} \sqrt[3]{-\frac{\beta_{1 n}}{2}+\sqrt{\frac{\beta_{1 n}^{2}}{4}+\frac{\alpha_{1 n}^{3}}{27}}}+\omega \sqrt[3]{-\frac{\beta_{1 n}}{2}-\sqrt{\frac{\beta_{1 n}^{2}}{4}+\frac{\alpha_{1 n}^{3}}{27}}},
\end{aligned}
$$

are the roots of the algebraic equations $X^{3}+\alpha_{1 n} X+\beta_{1 n}=0$, where

$$
\begin{aligned}
& \alpha_{1 n}=-\frac{\left(\lambda_{1}+\lambda_{4} v r_{n}^{2}\right)^{2}}{3 \lambda_{2}^{2}}+\frac{1+\lambda_{3} v r_{n}^{2}}{\lambda_{2}}, \\
& \beta_{1 n}=\frac{v r_{n}^{2}}{\lambda_{2}}+\frac{2\left(\lambda_{1}+\lambda_{4} v r_{n}^{2}\right)^{3}}{27 \lambda_{2}^{3}}-\frac{\left(\lambda_{1}+\lambda_{4} v r_{n}^{2}\right)\left(1+\lambda_{3} v r_{n}^{2}\right)}{3 \lambda_{2}^{2}},
\end{aligned}
$$


and

$$
\omega=\frac{-1+i \sqrt{3}}{2}
$$

From the Routh-Hurwitz's principle [19], we get $\operatorname{Re}\left(q_{i n}\right)<0$, if $\lambda_{1} \lambda_{3}-\lambda_{2}+\lambda_{4}>-2 \sqrt{\lambda_{1} \lambda_{3} \lambda_{4}}$, provided $\lambda_{1}, \lambda_{2}, \lambda_{3}, \lambda_{4}>0$.

\subsection{Calculation of the shear stress}

Applying the Laplace transform to Eq. (5), we find that

$$
\bar{\tau}(r, q)=\mu \frac{1+\lambda_{3} q+\lambda_{4} q^{2}}{1+\lambda_{1} q+\lambda_{2} q^{2}} \frac{\partial \bar{v}(r, q)}{\partial r},
$$

where

$$
\begin{aligned}
& \frac{\partial \bar{v}(r, q)}{\partial r}=\frac{R_{1} f}{\mu r} \frac{1}{q\left(1+\lambda_{3} q+\lambda_{4} q^{2}\right)}+\frac{\pi f}{\mu} \sum_{n=1}^{\infty} \frac{J_{0}^{2}\left(R_{2} r_{n}\right) \widetilde{B}\left(r, r_{n}\right)}{\left[J_{1}^{2}\left(R_{1} r_{n}\right)-J_{0}^{2}\left(R_{2} r_{n}\right)\right]} \\
& \times \frac{1+\lambda_{1} q+\lambda_{2} q^{2}}{\left(1+\lambda_{3} q+\lambda_{4} q^{2}\right)\left\{\lambda_{2} q^{3}+\left(\lambda_{1}+\lambda_{4} v r_{n}^{2}\right) q^{2}+\left(1+\lambda_{3} v r_{n}^{2}\right) q+v r_{n}^{2}\right\}},
\end{aligned}
$$

has been obtained from Eq. (18) applying the inverse Hankel transform and using Eqs. $(19)_{2}$ and (A2). Into above relation

$$
\widetilde{B}\left(r, r_{n}\right)=J_{1}\left(r r_{n}\right) Y_{1}\left(R_{1} r_{n}\right)-J_{1}\left(R_{1} r_{n}\right) Y_{1}\left(r r_{n}\right) .
$$

Finally, following the same way as in $[11,12]$, we find that

$$
\begin{gathered}
\tau(r, t)=f\left(\frac{R_{1}}{r}\right)\left[1-\frac{p_{2} e^{p_{1} t}-p_{1} e^{p_{2} t}}{p_{2}-p_{1}}\right]+\frac{\pi f}{\lambda_{2}} \sum_{n=1}^{\infty} \frac{J_{0}^{2}\left(R_{2} r_{n}\right) \widetilde{B}\left(r, r_{n}\right)}{J_{1}^{2}\left(R_{1} r_{n}\right)-J_{0}^{2}\left(R_{2} r_{n}\right)} \\
\times\left[\frac{e^{q_{1 n} t}}{\left(q_{1 n}-q_{2 n}\right)\left(q_{1 n}-q_{3 n}\right)}+\frac{e^{q_{3 n} t}}{\left(q_{2 n}-q_{1 n}\right)\left(q_{2 n}-q_{3 n}\right)}+\frac{\left.q_{1 n}\right)\left(q_{3 n}-q_{2 n}\right)}{\left(q_{3 n}-q_{1}\right)} .\right.
\end{gathered}
$$

By making $\lambda_{4} \rightarrow 0$ into Eqs. (20) and (24) the similar solutions for a Burgers' fluid performing the same motion are obtained. As a check of our results, let us remember the solutions [20, Eqs. (15) and (18)]

$$
v_{\mathrm{OB}}(r, t)=\frac{f}{\mu} R_{1} \ln \left(\frac{r}{R_{2}}\right)-\frac{\pi f}{\mu} \sum_{n=1}^{\infty} \frac{J_{0}^{2}\left(R_{2} r_{n}\right) B\left(r, r_{n}\right)}{r_{n}\left[J_{1}^{2}\left(R_{1} r_{n}\right)-J_{0}^{2}\left(R_{2} r_{n}\right)\right]} \frac{q_{5 n} e^{q_{4 n} t}-q_{4 n} e^{q_{5 n} t}}{q_{5 n}-q_{4 n}}
$$

and the shear stress

$$
\tau_{\mathrm{OB}}(r, t)=f\left(\frac{R_{1}}{r}\right)\left[1-e^{-t / \lambda_{1}}\right]+\frac{\pi f}{\lambda_{1}} \sum_{n=1}^{\infty} \frac{J_{0}^{2}\left(R_{2} r_{n}\right) \widetilde{B}\left(r, r_{n}\right)}{J_{1}^{2}\left(R_{1} r_{n}\right)-J_{0}^{2}\left(R_{2} r_{n}\right)} \frac{e^{q_{5 n} t}-e^{q_{4 n} t}}{q_{5 n}-q_{4 n}},
$$

corresponding to an Oldroyd-B fluid performing the same motion. These solutions correspond to the boundary condition

$$
\tau\left(R_{1}, t\right)=f\left(1-e^{-\frac{t}{\lambda_{1}}}\right),
$$


obtained form Eq. (8) for $\lambda_{2} \rightarrow 0$, and

$$
q_{4 n}, q_{5 n}=\frac{-\left(1+\lambda_{3} v r_{n}^{2}\right) \pm \sqrt{\left(1+\lambda_{3} v r_{n}^{2}\right)^{2}-4 v \lambda_{1} r_{n}^{2}}}{2 \lambda_{1}}
$$

By now letting $\lambda_{1}$ and $\lambda_{3} \rightarrow 0$ into Eqs. (25) and (26), the similar solutions (see [13, Eq. (4.35)]) with $\alpha=0$ for velocity only)

$$
\begin{aligned}
& v_{N}(r, t)=\frac{f}{\mu} R_{1} \ln \left(\frac{r}{R_{2}}\right)-\frac{\pi f}{\mu} \sum_{n=1}^{\infty} \frac{J_{0}^{2}\left(R_{2} r_{n}\right) B\left(r, r_{n}\right)}{r_{n}\left[J_{1}^{2}\left(R_{1} r_{n}\right)-J_{0}^{2}\left(R_{2} r_{n}\right)\right]} e^{-v r_{n}^{2} t}, \\
& \tau_{N}(r, t)=f\left(\frac{R_{1}}{r}\right)+\pi f \sum_{n=1}^{\infty} \frac{J_{0}^{2}\left(R_{2} r_{n}\right) \widetilde{B}\left(r, r_{n}\right)}{J_{1}^{2}\left(R_{1} r_{n}\right)-J_{0}^{2}\left(R_{2} r_{n}\right)} e^{-v r_{n}^{2} t},
\end{aligned}
$$

corresponding to Newtonian fluids are recovered. They correspond to a constant shear stress $\tau\left(R_{1}, t\right)=f$ on the inner cylinder.

Figures 1 and 2 clearly show that the diagram of $v(r, t)$ and $\tau(r, t)$ given by Eqs. (20) and (24), as it was to be expected, are almost identical to those corresponding to Oldroyd-B and Newtonian fluids for small values of $\lambda_{2}$ and $\lambda_{4}$, respectively $\lambda_{1}, \lambda_{2}, \lambda_{3}$, and $\lambda_{4}$. This is a veritable check on the corrections of general solutions presented in the simple forms (20) and (24).

\section{Steady and transient solutions}

The solutions (28) and (29), as well as those for second grade fluids resulting from Eqs. (25) and (26) for $\lambda_{1} \rightarrow 0$, correspond to a constant shear stress on the boundary. For large values of time $t$ these solutions tend to the steady solutions

$$
v_{S}(r)=\frac{f}{\mu} R_{1} \ln \left(\frac{r}{R_{2}}\right), \quad \tau_{S}(r)=f\left(\frac{R_{1}}{r}\right),
$$

which are the same for all types of fluids. Indeed, making $t \rightarrow \infty$ into Eqs. (20), (24), (25), and (26) we attain to Eqs. (30). This is not a surprise, because for large values of $t$ the boundary condition (8) is identical to that corresponding to second grade and

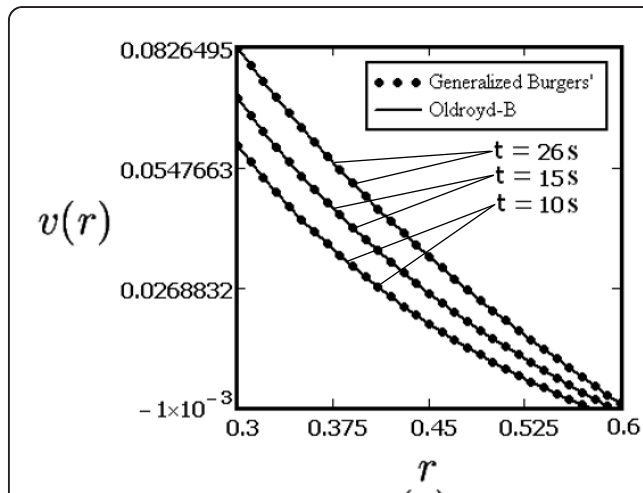

(a)

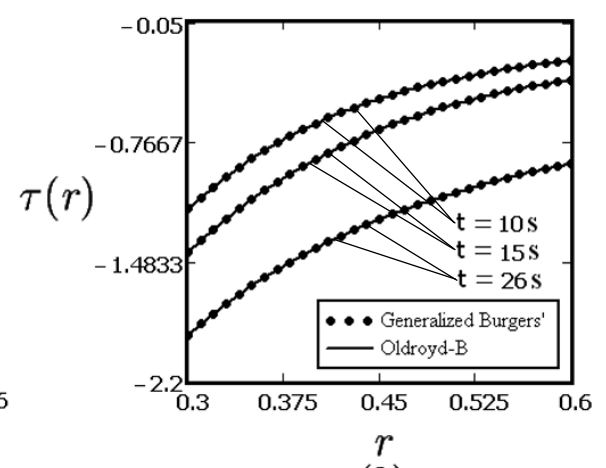

(b)

Figure 1 Profiles of the velocity $v(r, t)$ and the shear stress $\tau(r, t)$ for generalized Burgers and Oldroyd-B fluids, for $R_{1}=0.3, R_{2}=0.6, f=-2, v=0.005, \mu=5, \lambda_{1}=8, \lambda_{2}=0.00001, \lambda_{3}=4, \lambda_{4}=$ 0.00001 and different values of $t$. 


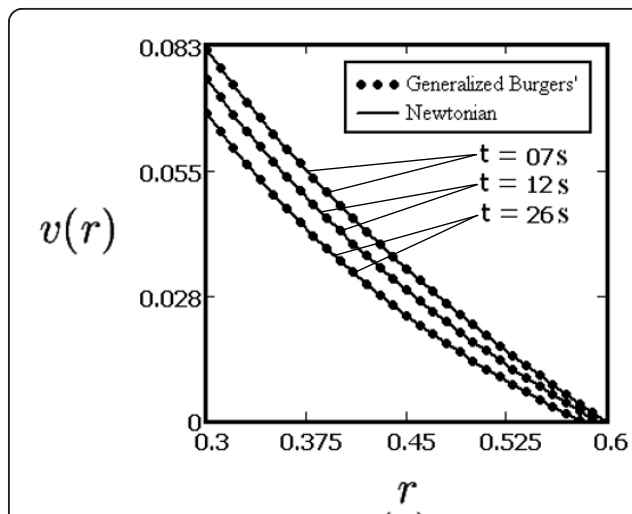

(a)

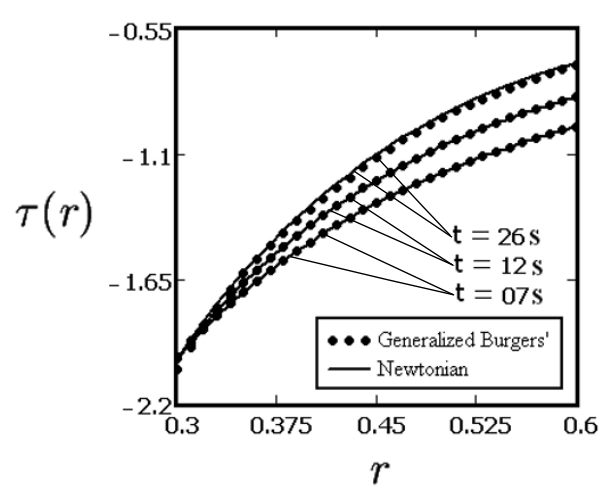

(b)

Figure 2 Profiles of the velocity $v(r, t)$ and the shear stress $\tau(r, t)$ for generalized Burgers and Newtonian fluids, for $R_{1}=0.3, R_{2}=0.6, f=-2, v=0.005, \mu=5, \lambda_{1}=0.00001, \lambda_{2}=0.00001, \lambda_{3}=$ $0.00001, \lambda_{4}=0.00001$ and different values of $t$.

Newtonian fluids. In conclusion, after some time, the behavior of a non-Newtonian fluid can be well enough described by that of a Newtonian fluid. This time, depending of the material parameters and the constant $f$, can easily be determined by graphical illustrations. In order to show this, the decay of the transient solutions

$$
\begin{gathered}
v_{T}(r, t)=\frac{\pi f}{\rho \lambda_{2}} \sum_{n=1}^{\infty} \frac{r_{n} J_{0}^{2}\left(R_{2} r_{n}\right) B_{v}\left(r, r_{n}\right)}{J_{1}^{2}\left(R_{1} r_{n}\right)-J_{0}^{2}\left(R_{2} r_{n}\right)} \\
\times\left[\frac{e^{q_{1 n} t}}{q_{1 n}\left(q_{1 n}-q_{2 n}\right)\left(q_{1 n}-q_{3 n}\right)}+\frac{e^{q_{3 n} t}}{q_{2 n}\left(q_{2 n}-q_{1 n}\right)\left(q_{2 n}-q_{3 n}\right)}+\frac{\tau^{q_{2 n}}\left(q_{3 n}-q_{1 n}\right)\left(q_{3 n}-q_{2 n}\right)}{q_{3 n}}\right], \\
\times\left[\frac{\tau_{T}(r, t)=f\left(\frac{R_{1}}{r}\right)\left[\frac{p_{1} e^{p_{2} t}-p_{2} e^{p_{1} t}}{p_{2}-p_{1}}\right]+\frac{\pi f}{\lambda_{2}} \sum_{n=1}^{\infty} \frac{J_{0}^{2}\left(R_{2} r_{n}\right) \widetilde{B}_{v}\left(r, r_{n}\right)}{J_{1}^{2}\left(R_{1} r_{n}\right)-J_{0}^{2}\left(R_{2} r_{n}\right)}}{\left(q_{1 n}-q_{2 n}\right)\left(q_{1 n}-q_{3 n}\right)}+\frac{e^{q_{2 n} t}}{\left(q_{2 n}-q_{1 n}\right)\left(q_{2 n}-q_{3 n}\right)}+\frac{e^{q_{3 n} t}}{\left(q_{3 n}-q_{1 n}\right)\left(q_{3 n}-q_{2 n}\right)}\right],
\end{gathered}
$$

in time, is depicted in Figures 3, 4, and 5 for different values of $\lambda_{1}, \lambda_{3}$ and $v$. These figures clearly show that the required time to reach the steady state is an increasing

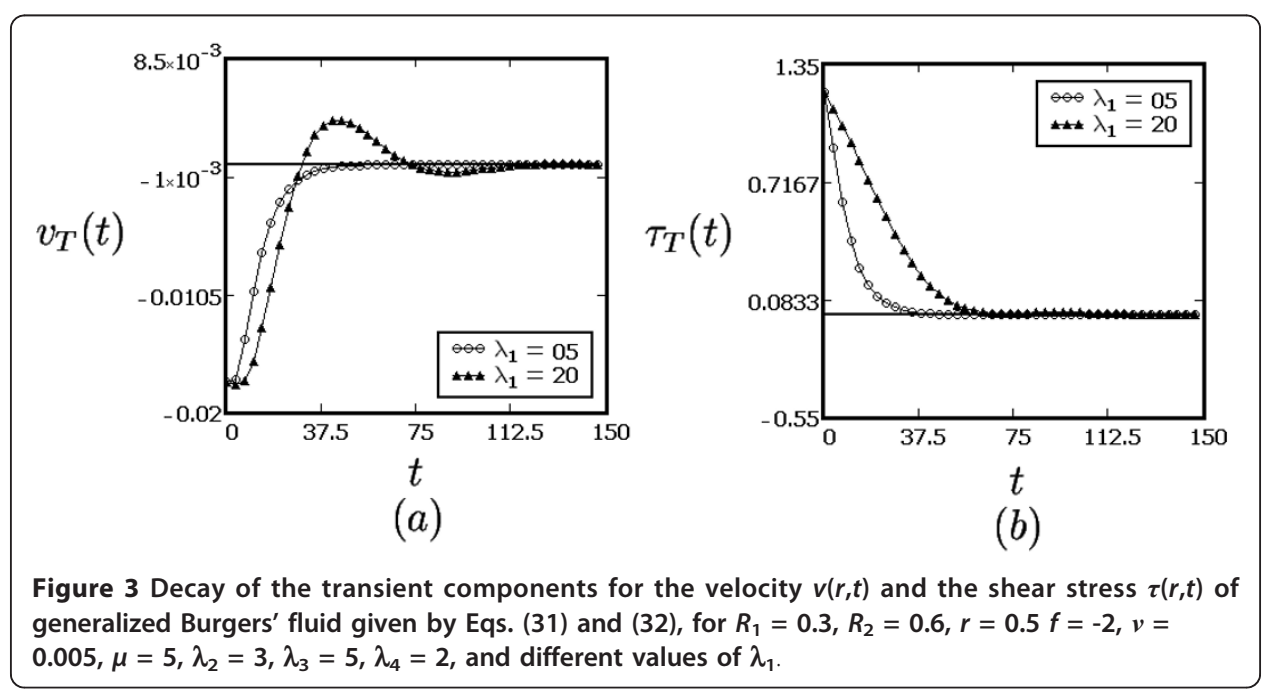




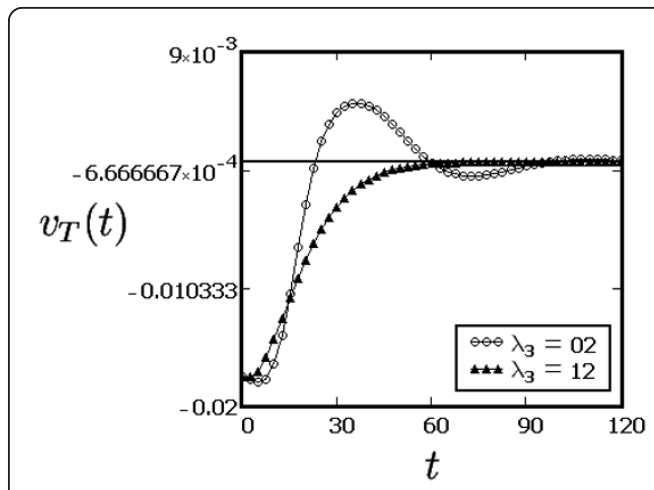

$(a)$

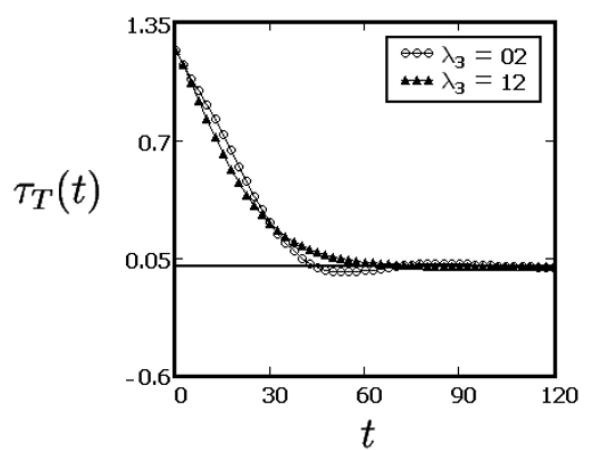

(b)

Figure 4 Decay of the transient components for the velocity $v(r, t)$ and the shear stress $\tau(r, t)$ of generalized Burgers' fluid given by Eqs. (31) and (32), for $R_{1}=0.3, R_{2}=0.6, r=0.5 f=-2, v=$ $0.005, \mu=5, \lambda_{1}=15, \lambda_{2}=5, \lambda_{4}=2$, and different values of $\lambda_{3}$.

function with respect to $\lambda_{1}$ and a decreasing one with regard to $\lambda_{3}$ and $v$. A simple analysis shows that the required time to reach the steady state for Maxwell fluids, for instance, is higher in comparison with Newtonian fluids. According to Figure 4, it also results that the required time to reach the steady state is lower for second grade fluids in comparison with Newtonian fluids.

\section{Numerical results and discussion}

In the previous sections, we have presented exact analytical solutions for a flow problem of a generalized Burgers' fluid. In order to verify and capture relevant physical effects of the obtained results, several graphs are depicted in this section. The numerical results illustrate the velocity as well as shear stress profiles for the axial flow induced by the inner cylinder. We interpret these results with respect to the variations of emerging parameters of interest.

For large values of $t$, all solutions tend to the steady solutions $v_{S}(r, t)$ and $\tau_{S}(r, t)$ which are the same for all kinds of fluids although the motion of rate type fluids (generalized Burgers, Burgers, Oldroyd-B, and Maxwell) is due to a time dependent shear stress on

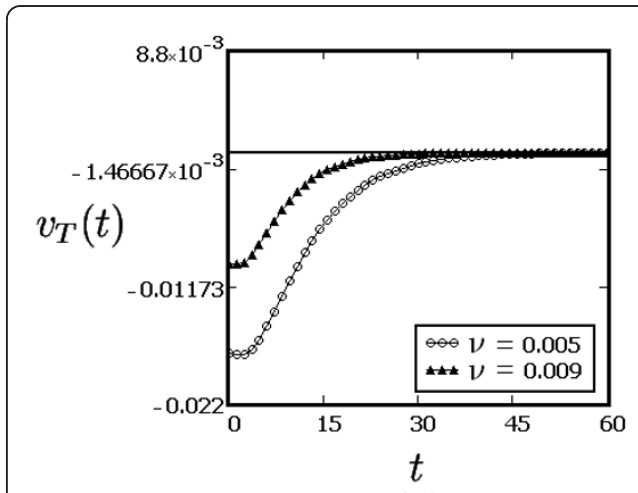

(a)

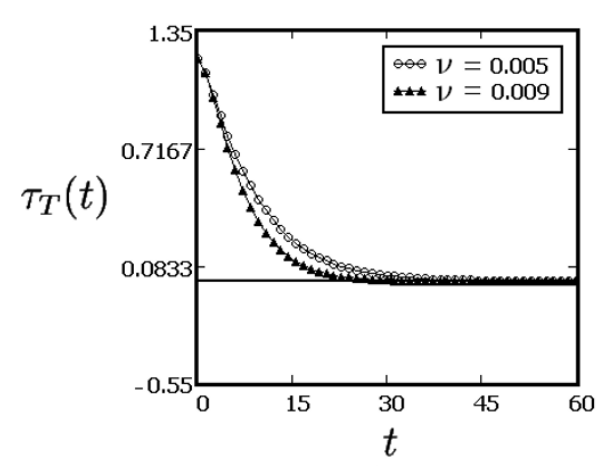

(b)

Figure 5 Decay of the transient components for the velocity $v(r, t)$ and the shear stress $\tau(r, t)$ of generalized Burgers' fluid given by Eqs. (31) and (32), for $R_{1}=0.3, R_{2}=0.6, r=0.5 f=-2, \rho=$ $1000, \lambda_{1}=5, \lambda_{2}=3, \lambda_{3}=5, \lambda_{4}=2$ and different values of $v$. 
the boundary. However, this is not a surprise because, for large times, the boundary shear stress corresponding to rate type fluids, as it results from Eqs. (8) and (29), tends to the same constant $f$ corresponding to Newtonian and second grade fluids.

From Figures 1 and 2 it also results that both the velocity and the shear stress (in absolute value) are increasing functions with respect to $t$. Moreover, they are decreasing functions with respect to $r$. Figures 6 and 7 are prepared to show the influence of the relaxation time $\lambda_{1}$ and the rheological parameter, $\lambda_{2}$ on the fluid motion. The two parameters, as expected, have opposite effects on the fluid motion. Both the velocity field and the shear stress (in absolute value) are decreasing functions with respect to relaxation time $\lambda_{1}$ and increasing ones with respect to $\lambda_{2}$ on the whole flow domain. Figures 8 and 9 are depicted to show the effects of retardation time $\lambda_{3}$ and rheological parameter $\lambda_{4}$ on the fluid motion. It is observed that greater the retardation time $\lambda_{3}$, the more rapidly decay the velocity, however the shear stress is grown up faster. The influence of the rheological parameter $\lambda_{4}$ on the fluid motion is shown by Figure 9. Its effect is opposite to that of $\lambda_{3}$. The influence of the kinematic viscosity $v$ on the fluid motion is shown in Figure 10. From these figures, we can see that the velocity decreases and the shear stress increases by increasing $v$.

Finally, for comparison, the profiles of velocity and shear stress corresponding to the motion of the three types of fluids (Newtonian, Oldroyd-B and generalized Burgers) are together depicted in Figure 11 for different values of $t$ and of common material parameters. The Newtonian fluid, as it results from Figure 11a1, is the swiftest and the generalized Burgers' fluid is the slowest on the whole flow domain. The shear stress corresponding to the Newtonian fluid in absolute value is also higher on the whole flow domain. Moreover, Figure 11-a2,b2,a3,b3 show that for large values of $t$, the velocity as well as the shear stress corresponding to generalized Burgers and Oldryd-B fluids, as it was to be expected, are going to those belonging to a Newtonian fluid. Consequently, the non-Newtonian effects disappear in time. The units of the material constants are SI units in all figures and the roots $r_{n}$ have been approximated by $(2 n$ 1) $\pi /\left[2\left(R_{2}-R_{1}\right)\right]$.

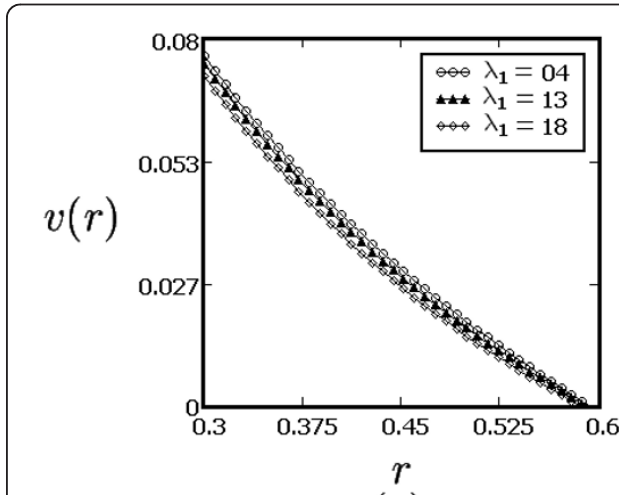

(a)

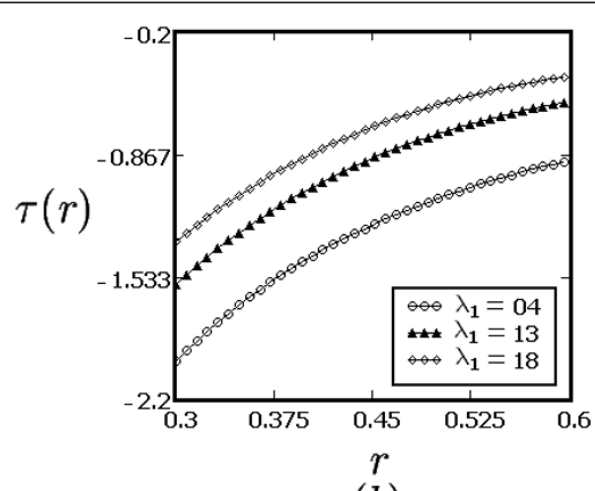

(b)

Figure 6 Profiles of the velocity $v(r, t)$ and the shear stress $\tau(r, t)$ given by Eqs. (20) and (24), for $R_{1}=$ $0.3, R_{2}=0.6, f=-2, v=0.005, \mu=5, \lambda_{2}=3, \lambda_{3}=5, \lambda_{4}=2, t=20$ s and different values of $\lambda_{1}$. 


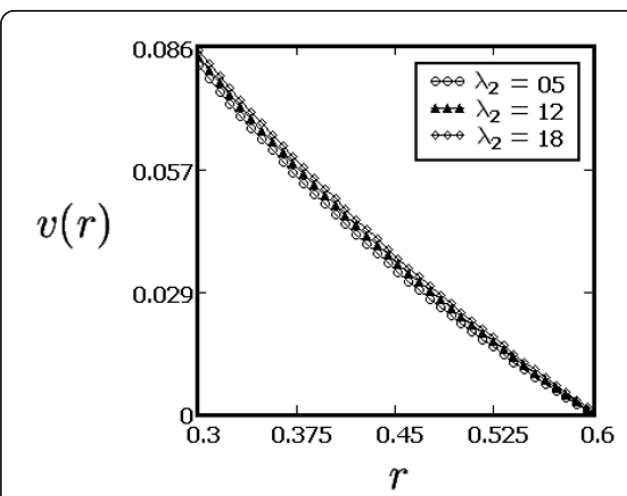

(a)

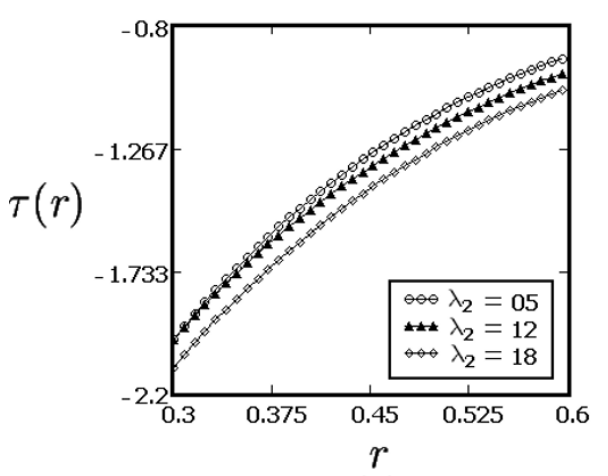

(b)

Figure 7 Profiles of the velocity $v(r, t)$ and the shear stress $\tau(r, t)$ given by Eqs. (20) and (24), for $R_{1}=$ $0.3, R_{2}=0.6, f=-2, v=0.005, \mu=5, \lambda_{1}=3, \lambda_{3}=2, \lambda_{4}=12, t=20 \mathrm{~s}$ and different values of $\lambda_{2}$.

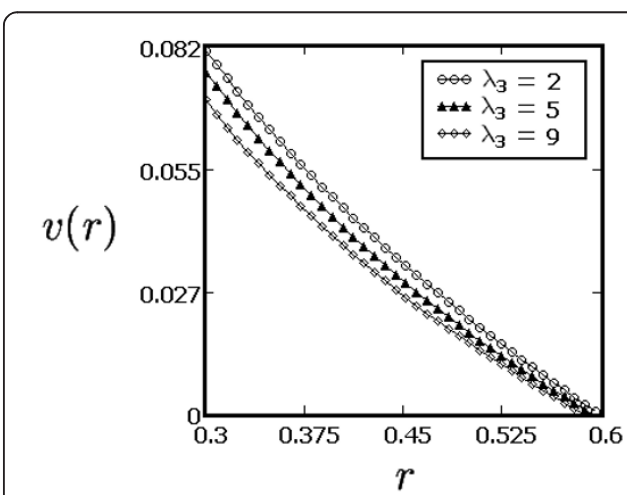

(a)

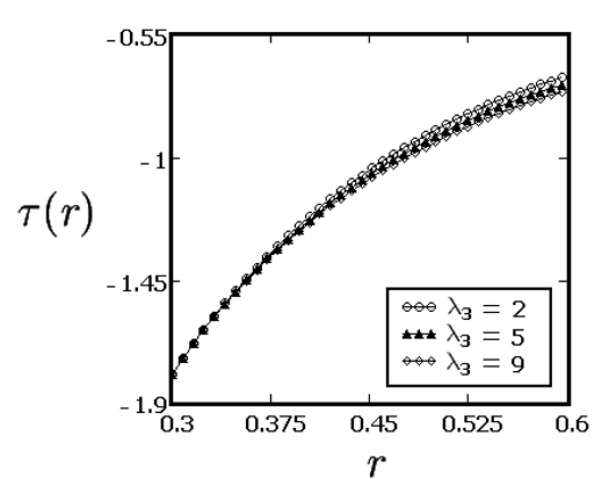

(b)

Figure 8 Profiles of the velocity $v(r, t)$ and the shear stress $\tau(r, t)$ given by Eqs. (20) and (24), for $R_{1}=$ $0.3, R_{2}=0.6, f=-2, v=0.005, \mu=5, \lambda_{1}=9, \lambda_{2}=3, \lambda_{4}=2, t=20 \mathrm{~s}$ and different values of $\lambda_{3}$.

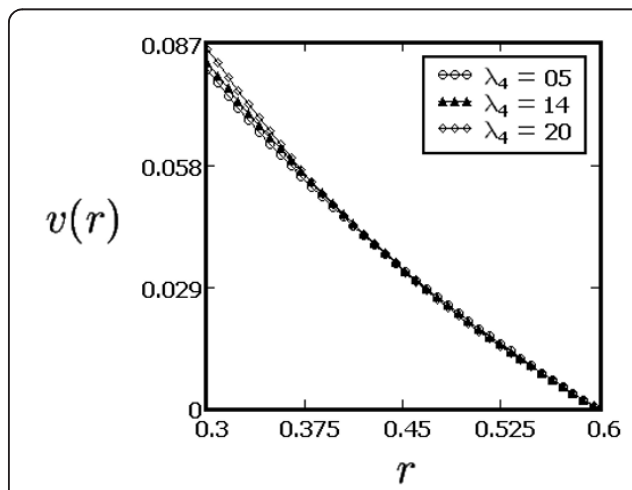

(a)

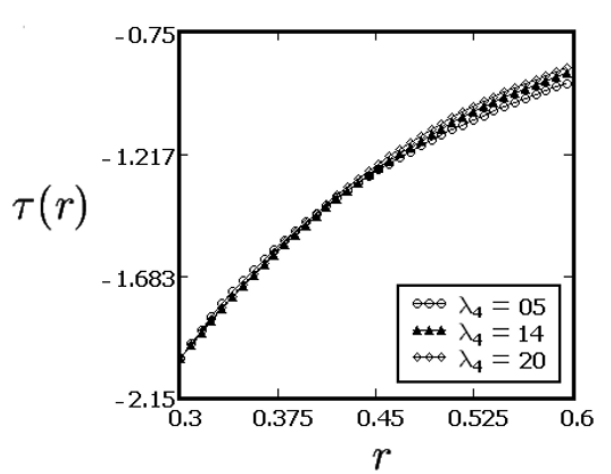

(b)

Figure 9 Profiles of the velocity $v(r, t)$ and the shear stress $\tau(r, t)$ given by Eqs. (20) and (24), for $R_{1}$ $=0.3, R_{2}=0.6, f=-2, v=0.005, \mu=5, \lambda_{1}=3, \lambda_{2}=3, \lambda_{3}=2, t=20 \mathrm{~s}$ and different values of $\lambda_{4}$. 


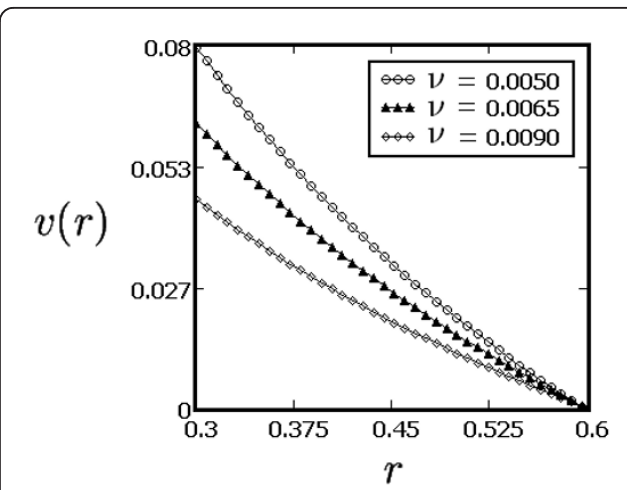

(a)

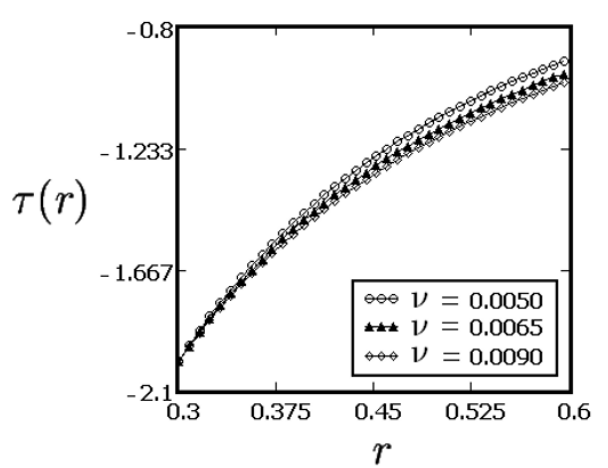

(b)

Figure 10 Profiles of the velocity $v(r, t)$ and the shear stress $\tau(r, t)$ given by Eqs. (20) and (24), for $R_{1}$ $=0.3, R_{2}=0.6, f=-2, \rho=1000, \lambda_{1}=4, \lambda_{2}=3, \lambda_{3}=3, \lambda_{4}=2, t=20 \mathrm{~s}$ and different values of $v$.

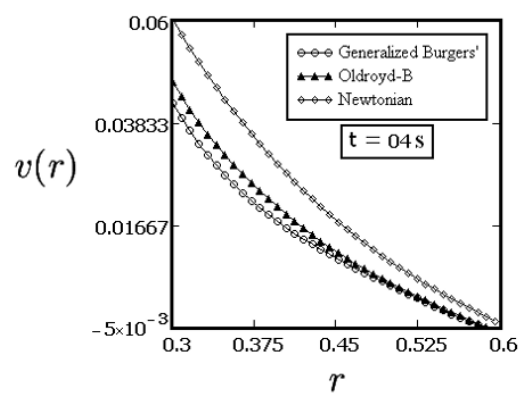

$(a 1)$

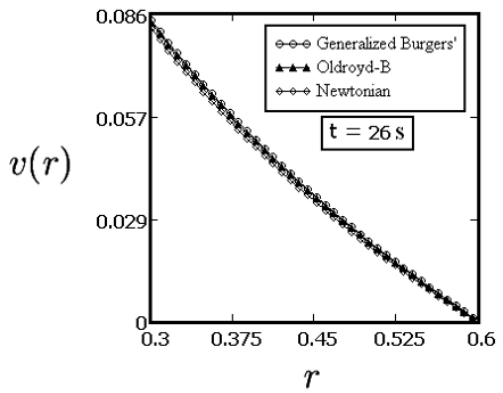

$(a 2)$

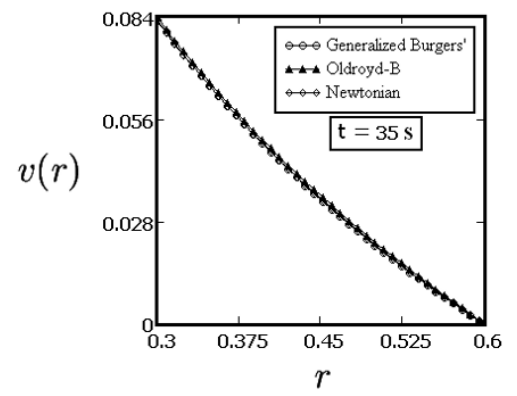

$(a 3)$

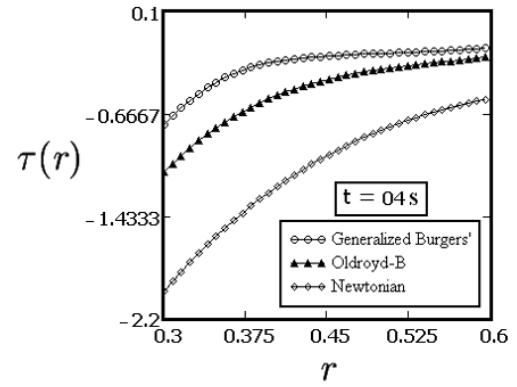

$(b 1)$

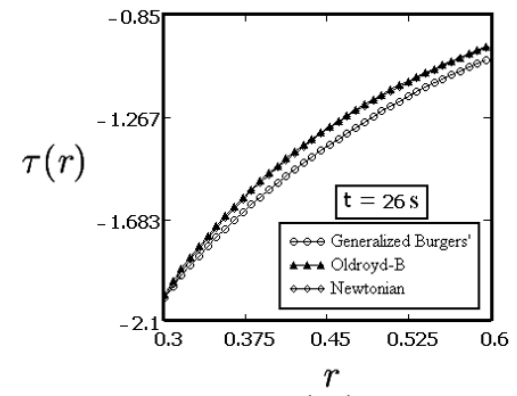

$(b 2)$

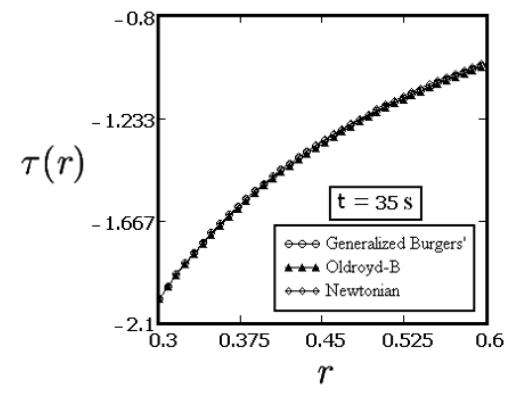

$(b 3)$

Figure 11 Profiles of the velocity $v(r, t)$ and the shear stress $\tau(r, t)$ for generalized Burgers', Oldroyd$B$ and Newtonian fluids, for $R_{1}=0.3, R_{2}=0.6, f=-2, v=0.005, \mu=5, \lambda_{1}=5, \lambda_{2}=12, \lambda_{3}=2, \lambda_{4}=$ 2 and different values of $t$. 


\section{Concluding remarks}

In this article, the velocity $v(r, t)$ and the shear stress $\tau(r, t)$ corresponding to the flow of an incompressible generalized Burgers' fluid, between two infinite coaxial circular cylinders, have been determined by means of Laplace and finite Hankel transforms. The motion of the fluid is produced by the inner cylinder that, after the initial moment, applies a time dependent longitudinal shear to the fluid. The solutions that have been obtained, written in series form in terms of Bessel functions $J_{0}(\bullet), J_{1}(\bullet), Y_{0}(\bullet)$, and $Y_{1}(\bullet)$, satisfy all imposed initial and boundary conditions. They can easily be reduced to the similar solutions for Burgers fluids. For large values of $t$, all solutions are going to the steady solutions $v_{S}(r)$ and $\tau_{S}(r)$, which are the same for all kinds of fluids. The following conclusions may be extracted from graphical results.

- The required time to reach the steady-state increases with respect to $\lambda_{1}$ and decreases with regard to $\lambda_{3}$ and $v$. Consequently, the required time to reach the steady state for Newtonian fluids is lower/higher in comparison with Maxwell, respectively, second grade fluids.

- For small values of $\lambda_{3}$ and $\lambda_{4}$ or $\lambda_{1}, \lambda_{2}, \lambda_{3}$, and $\lambda_{4}$ the general solutions (20) and (24), as expected, are equivalent to those corresponding to Oldroyd-B, respectively, Newtonian fluids.

- The relaxation parameters $\lambda_{1}$ and $\lambda_{2}$, as well as the retardation parameters $\lambda_{3}$ and $\lambda_{4}$, have opposite effects on the fluid motion. More exactly, the velocity $v(r, t)$ and the shear stress $\tau(r, t)$ in absolute value are decreasing functions with respect to $\lambda_{1}$ and $\lambda_{3}$ and increasing ones with regard to $\lambda_{2}$ and $\lambda_{4}$.

- It is observed that the increase of the kinematic viscosity $v$ leads to a decay of velocity and grown up the shear stress.

- The Newtonian fluid is the swiftest and the generalized Burgers' fluid is the slowest.

- The non-Newtonian effects disappear in time.

\section{Appendix}

$$
\left.\begin{array}{l}
\mathcal{L}^{-1}\left\{\frac{\lambda_{2} q^{2}+\left(\lambda_{1}+\lambda_{4} v r_{n}^{2}\right) q+1+\lambda_{3} v r_{n}^{2}}{\lambda_{2} q^{3}+\left(\lambda_{1}+\lambda_{4} v r_{n}^{2}\right) q^{2}+\left(1+\lambda_{3} v r_{n}^{2}\right) q+v r_{n}^{2}}\right\} \\
=\frac{1}{\lambda_{2}} \mathcal{L}^{-1}\left\{\frac{\lambda_{2} q^{2}+\left(\lambda_{1}+\lambda_{4} v r_{n}^{2}\right) q+1+\lambda_{3} v r_{n}^{2}}{\left(q-q_{1 n}\right)\left(q-q_{2 n}\right)\left(q-q_{3 n}\right)}\right\} \\
=\frac{1}{\lambda_{2}} \mathcal{L}^{-1}\left\{\frac{\lambda_{2} q_{1 n}^{2}+\left(\lambda_{1}+\lambda_{4} v r_{n}^{2}\right) q_{1 n}+1+\lambda_{3} v r_{n}^{2}}{\left(q_{1 n}-q_{2 n}\right)\left(q_{1 n}-q_{3 n}\right)\left(q-q_{1 n}\right)}+\frac{\lambda_{2} q_{2 n}^{2}+\left(\lambda_{1}+\lambda_{4} v r_{n}^{2}\right) q_{2 n}+1+\lambda_{3} v r_{n}^{2}}{\left(q_{2 n}-q_{1 n}\right)\left(q_{2 n}-q_{3 n}\right)\left(q-q_{2 n}\right)}\right. \\
\left.\quad+\frac{\lambda_{2} q_{3 n}^{2}+\left(\lambda_{1}+\lambda_{4} v r_{n}^{2}\right) q_{3 n}+1+\lambda_{3} v r_{n}^{2}}{\left(q_{3 n}-q_{1 n}\right)\left(q_{3 n}-q_{2 n}\right)\left(q-q_{3 n}\right)}\right\}
\end{array}\right\}
$$




$$
\begin{aligned}
& \mathcal{L}^{-1}\left\{\frac{1}{q\left(\lambda_{2} q^{2}+\lambda_{1} q+1\right)}\right\} \\
& =\frac{1}{\lambda_{2}} \mathcal{L}^{-1}\left\{\frac{1}{q\left(q-p_{1}\right)\left(q-p_{2}\right)}\right\} \\
& =\frac{1}{\lambda_{2}} \mathcal{L}^{-1}\left\{\frac{1}{p_{1} p_{2} q}+\frac{1}{p_{1}\left(p_{1}-p_{2}\right)\left(q-p_{1}\right)}+\frac{1}{p_{2}\left(p_{2}-p_{1}\right)\left(q-p_{2}\right)}\right\} \\
& =1-\frac{p_{2} e^{p_{1} t}-p_{1} e^{p_{2} t}}{p_{2}-p_{1}} \\
& \mathcal{L}^{-1}\left\{\frac{1}{\lambda_{2} q^{3}+\left(\lambda_{1}+\lambda_{4} v r_{n}^{2}\right) q^{2}+\left(1+\lambda_{3} v r_{n}^{2}\right) q+v r_{n}^{2}}\right\} \\
& =\frac{1}{\lambda_{2}} \mathcal{L}^{-1}\left\{\frac{1}{\left(q-q_{1 n}\right)\left(q-q_{2 n}\right)\left(q-q_{3 n}\right)}\right\} \\
& =\frac{1}{\lambda_{2}} \mathcal{L}^{-1}\left\{\frac{1}{\left(q_{1 n}-q_{2 n}\right)\left(q_{1 n}-q_{3 n}\right)\left(q-q_{1 n}\right)}+\frac{1}{\left(q_{2 n}-q_{1 n}\right)\left(q_{2 n}-q_{3 n}\right)\left(q-q_{2 n}\right)}\right. \\
& \left.+\frac{1}{\left(q_{3 n}-q_{1 n}\right)\left(q_{3 n}-q_{2 n}\right)\left(q-q_{3 n}\right)}\right\} \\
& =\frac{1}{\lambda_{2}}\left\{\frac{e^{q_{1 n} t}}{\left(q_{1 n}-q_{2 n}\right)\left(q_{1 n}-q_{3 n}\right)}+\frac{e^{q_{2 n} t}}{\left(q_{2 n}-q_{1 n}\right)\left(q_{2 n}-q_{3 n}\right)}\right. \\
& \left.+\frac{e^{q_{3 n} t}}{\left(q_{3 n}-q_{1 n}\right)\left(q_{3 n}-q_{2 n}\right)}\right\} \\
& \mathcal{L}^{-1}\left\{\frac{\lambda_{1} q+1+\lambda_{3} v r_{n}^{2}}{\lambda_{1} q^{2}+\left(1+\lambda_{3} v r_{n}^{2}\right) q+v r_{n}^{2}}\right\} \\
& =\frac{1}{\lambda_{1}} \mathcal{L}^{-1}\left\{\frac{\lambda_{1} q+1+\lambda_{3} v r_{n}^{2}}{\left(q-q_{7}\right)\left(q-q_{8}\right)}\right\} \\
& =\frac{1}{\lambda_{1}} \mathcal{L}^{-1}\left\{\frac{\lambda_{1} q_{1 n}+1+\lambda_{3} v r_{n}^{2}}{\left(q_{7 n}-q_{8 n}\right)\left(q-q_{7 n}\right)}+\frac{\lambda_{1} q_{2}+1+\lambda_{3} v r_{n}^{2}}{\left(q_{8 n}-q_{7 n}\right)\left(q-q_{8 n}\right)}\right\} \\
& =\frac{q_{8 n} e^{q_{7 n} t}-q_{7 n} e^{q_{8 n} t}}{q_{8 n}-q_{7 n}} \\
& \mathcal{L}^{-1}\left\{\frac{1}{q\left(1+\lambda_{1} q\right)}\right\}=\mathcal{L}^{-1}\left\{\frac{1}{q}-\frac{\lambda_{1}}{1+\lambda_{1} q}\right\}=1-e^{-\frac{t}{\lambda_{1}}} \\
& \mathcal{L}^{-1}\left\{\frac{1}{\lambda_{1} q^{2}+\left(1+\lambda_{3} v r_{n}^{2}\right) q+v r_{n}^{2}}\right\} \\
& =\frac{1}{\lambda_{1}} \mathcal{L}^{-1}\left\{\frac{1}{\left(q-q_{7 n}\right)\left(q-q_{8 n}\right)}\right\} \\
& =\frac{1}{\lambda_{1}} \mathcal{L}^{-1}\left\{\frac{-1}{\left(q_{8 n}-q_{7 n}\right)\left(q-q_{7 n}\right)}+\frac{1}{\left(q_{8 n}-q_{7 n}\right)\left(q-q_{8 n}\right)}\right\} \\
& =\frac{1}{\lambda_{1}} \frac{e^{q_{8 n} t}-e^{q_{7 n} t}}{q_{8 n}-q_{7 n}} \text {. }
\end{aligned}
$$

\section{Acknowledgements}

The authors would like to express their sincere gratitude to the referees for their careful assessment and fruitful remarks and suggestions regarding the initial version of the manuscript. The author Muhammad Jamil highly thankful 
and grateful to the Abdus Salam School of Mathematical Sciences, GC University, Lahore, Pakistan; Department of Mathematics, NED University of Engineering \& Technology, Karachi-75270, Pakistan and also Higher Education Commission of Pakistan for generous supporting and facilitating this research study.

\section{Author details}

${ }^{1}$ Abdus Salam School of Mathematical Sciences, GC University, Lahore, Pakistan ${ }^{2}$ Department of Mathematics, NED University of Engineering \& Technology, Karachi-75270, Pakistan ${ }^{3}$ Department of Mathematics, Technical University of lasi, lasi-700050, Associate member of Academy of Romanian Scientists, 050094 Bucarest, Romania

\section{Authors' contributions}

Qualitatively, the authors' contribution for this paper is as follows: MJ (Muhammad Jamil) 60\% and CF (Constantin Fetecau) $40 \%$. Both authors participed in the design of the study and performed the statistical analysis. MJ has had a special contribution to get the simplest form of solutions. CF participated in the sequence alignment and drafted the manuscript. MJ prepared all graphical illustrations and CF participated at conclusions and discussion. Both authors read and approved the final manuscript.

\section{Competing interests}

The authors declare that they have no competing interests.

Received: 28 March 2011 Accepted: 10 February 2012 Published: 10 February 2012

\section{References}

1. Burgers, JM: Mechanical considerations-model systems-phenomenological theories of relaxation and of viscosity. In: Burgers JM (ed.) First Report on Viscosity and Plasticity. Nordemann Publishing Company, New York (1935)

2. Krishnan, JM, Rajagopal, KR: A thermodynamic framework for the constitutive modeling of asphalt concrete: Theory and application. J Mater Civ Eng. 16, 155-166 (2004). doi:10.1061/(ASCE)0899-1561(2004)16:2(155)

3. Lee, AR, Markwick, AHD: The mechanical properties of bituminous surfacing materials under constant stress. J Soc Chem Ind. 56, 146 (1937)

4. Tan, BH, Jackson, I, Gerald, JDF: High-temperature viscoelasticity of fine-grained polycrystalline olivine. Phys Chem Miner. 28, 641 (2001). doi:10.1007/s002690100189

5. Peltier, WR, Wu, P, Yuen, DA: The viscosities of the earth mantle. In: Stacey, FD, Paterson, MS, Nicholas, A (eds.) Anelasticity in the Earth. American Geophysical Union, Colorado (1981)

6. Ravindran, P, Krishnan, JM, Rajagopal, KR: A note on the flow of a Burgers' fluid in an orthogonal rheometer. Internat J Eng Sci. 42, 1973-1985 (2004). doi:10.1016/j.jiengsci.2004.07.007

7. Khan, M, Ali, SH, Fetecau, C: Exact solutions of accelerated flows for a Burgers' fluid. I. The case $\gamma<\lambda^{2} / 4$. Appl Math Comput. 203, 881-894 (2008). doi:10.1016/j.amc.2008.05.121

8. Hayat, T, Khan, SB, Khan, M: Influence of Hall current on the rotating flow of a Burgers' fluid through a porous space. J Porous Med. 11, 277-287 (2008)

9. Tong, DK, Shan, LT: Exact solution for generalized Burgers' fluid in an annular pipe. Meccanica. 44, 427-431 (2009). doi:10.1007/s11012-008-9179-6

10. Fetecau, C, Hayat, T, Khan, M, Fetecau, C: A note on longitudinal oscillations of a generalized Burgers fluid in cylindrical domains. J Non-Newtonian Fluid Mech. 165, 350-361 (2010). doi:10.1016/j.jnnfm.2010.01.009

11. Jamil, M, Fetecau, C: Some exact solutions for rotating flows of a generalized Burgers' fluid in cylindrical domains. J Non-Newtonian Fluid Mech. 165, 1700-1712 (2010). doi:10.1016/j.jnnfm.2010.08.004

12. Jamil, M, Zafar, AA, Fetecau, C, Khan, NA: Exact analytic solutions for the flow of a generalized burgers fluid induced byan accelerated shear stress. Chem Eng Commun. 199, 17-39 (2012). doi:10.1080/00986445.2011.570389

13. Bandelli, R, Rajagopal, KR: Start-up flows of second grade fluids in domains with one finite dimension. Int J Non-Linear Mech. 30, 817-839 (1995). doi:10.1016/0020-7462(95)00035-6

14. Rajagopal, KR, Bhatnagar, RK: Exact solutions for some simple flows of an Oldroyd-B fluid. Acta Mech. 113, 223-239 (1995)

15. Tong, DK, Wang, RH, Yang, HS: Exact solutions for the flow of non-Newtonian fluid with fractional derivative in an annular pipe. Sci China Ser G. 48, 485-495 (2005)

16. Debnath, L, Bhatta, D: Integral Transforms and Their Applications, (second ed.). Chapman and Hall/CRC Press, BocaRaton-London-New York (2007)

17. Fetecau, C, Mahmood, A, Jamil, M: Exact solutions for the flow of a viscoelastic fluid induced by a circular cylinder subject to a time dependent shear stress. Commun Nonlinear Sci Numer Simulat. 15, 3931-3938 (2010). doi:10.1016/j. cnsns.2010.01.012

18. Kuros, A: Cours d'algebre superieure. Edition Mir Moscow (1973)

19. Morris, M: Geometry of Polynomials, Hayden stacks QA331.M322. (1966)

20. Corina, Fetecau, Awan, AU, Nazish, Shahid: Axial Coutte flow of an Oldroyd-B fluid in an annulus due to a timedependent shear stress. Bull Inst Polit lasi Tome LVII (LXI), Fasc. 4, 13-25 (2011)

doi:10.1186/1687-2770-2012-14

Cite this article as: Jamil and Fetecau: Starting solutions for the motion of a generalized Burgers' fluid between coaxial cylinders. Boundary Value Problems 2012 2012:14. 